\title{
Changes in Neuroticism Following Trauma Exposure
}

Journal of Personality $\cdot \cdot \cdot \cdot \bullet, \cdot \bullet 2013$

(C) 2013 Wiley Periodicals, Inc.

DOI: 10.1 I I I/jopy. 12037

\author{
Christin M. Ogle,' David C. Rubin,, ${ }^{1,2}$ and Ilene C. Siegler ${ }^{1,3}$ \\ 'Duke University \\ ${ }^{2}$ Center for Autobiographical Memory Research, Aarhus University \\ ${ }^{3}$ Duke University School of Medicine
}

\begin{abstract}
Using longitudinal data, the present study examined change in midlife neuroticism following trauma exposure. Our primary analyses included 670 participants $\left(M_{\text {age }}=60.55 ; 65.22 \%\right.$ male, $99.70 \%$ Caucasian $)$ who completed the NEO Personality Inventory at ages 42 and 50 and reported their lifetime exposure to traumatic events approximately 10 years later. No differences in pre- and post-trauma neuroticism scores were found among individuals who experienced all of their lifetime traumas in the interval between the personality assessments. Results were instead consistent with normative age-related declines in neuroticism throughout adulthood. Furthermore, longitudinal changes in neuroticism scores did not differ between individuals with and without histories of midlife trauma exposure. Examination of change in neuroticism following lifethreatening traumas yielded a comparable pattern of results. Analysis of facet-level scores largely replicated findings from the domain scores. Overall, our findings suggest that neuroticism does not reliably change following exposure to traumatic events in middle adulthood. Supplemental analyses indicated that individuals exposed to life-threatening traumas in childhood or adolescence reported higher midlife neuroticism than individuals who experienced severe traumas in adulthood. Lifethreatening traumatic events encountered early in life may have a more pronounced impact on adulthood personality than recent traumatic events.
\end{abstract}

Robust associations between elevated levels of neuroticism and exposure to traumatic events have been reported. Although, prospective studies concerning the impact of personality on trauma exposure indicate that higher levels of neuroticism increase the risk of experiencing traumatic life events (Breslau, Davis, Andreski, \& Peterson, 1995; Magnus, Diener, Fujita, \& Pavot, 1993; Specht, Egloff, \& Schmukle, 2011) as well as the severity of post-traumatic stress disorder (PTSD; Parslow, Jorm, \& Christensen, 2006; Rubin, Berntsen, \& Bohni, 2008), an alternative interpretation of the positive relation between neuroticism and traumatic events is that exposure to trauma leads individuals to become more neurotic. Support for this hypothesis is drawn from the few existing studies concerning changes in personality following adverse life events (e.g., Löckenhoff, Terracciano, Patriciu, Eaton, \& Costa, 2009). However, the majority of this research has examined the effects on personality of a wide range of negative and stressful events rather than the effects of traumatic events capable of producing PTSD symptoms. Furthermore, in this literature, personality changes are measured in individuals who experience adverse events during a relatively brief time interval, without consideration of their lifetime exposure to such events (e.g., Vaidya, Gray, Haig, \& Watson, 2002). It is possible that the pre-event (i.e., baseline) personality assessments in these studies were influenced by individuals' previous exposure to negative or traumatic experiences. The present study extends prior research by assessing personality before and after individuals' lifetime exposure to traumatic events, thereby providing a more conservative investigation of change in neuroticism following traumatic events.

Several opposing theoretical views of personality development can be used to inform predictions concerning continuity and change in neuroticism following exposure to traumatic events. According to the essentialist model (McCrae et al., 2000), personality traits are biologically determined and therefore are not susceptible to change as a result of environmental factors. Proponents of this view conclude that personality change occurs primarily during young adulthood and reaches a plateau by late middle age (McCrae et al., 1999). In contrast, various contextual perspectives (e.g., Lewis, 2001; Neyer \& Asendorpf, 2001) assert that personality traits are sensitive to

This study was funded in part by the National Institute on Aging (ROI-AGI2458 and 5T32 AG000029-35), the National Heart Lung and Blood Institute (ROI-HL55356 and POI-HL36587), the National Institute of Mental Health (ROI-MH066079), and the Duke Behavioral Medicine Research Center.

Correspondence concerning this article should be addressed to Christin M. Ogle, Department of Psychology \& Neuroscience, Duke University, Box 90086, Durham, NC 27708. Email: christin.ogle@duke.edu. 
environmental influences and are therefore likely to change over time and across contexts, especially during developmental periods characterized by pervasive internal and external change. More moderate and integrated variants of these two perspectives have also been proposed (e.g., Helson, Kwan, John, \& Jones, 2002; Kandler, 2012), according to which changes in personality occur throughout the life course and can be attributed to both genetic and environmental influences. Another relevant theoretical model of personality coherence (Caspi \& Moffitt, 1991, 1993) assumes that stressful life experiences do not exert fundamental and widespread transformations in personality, but rather preexisting individual differences in personality traits are magnified and accentuated during an individual's adaptation to challenging life events, especially unstructured events characterized by novelty, ambiguity, and uncertainty. Although the present study was not designed to directly test these diverse perspectives, our results may have implications for theoretical models of personality continuity and change given that the majority of adults report exposure to one or more traumatic events during their lifetime (Kessler, Sonnega, Bromet, Hughes, \& Nelson, 1995).

Data were drawn from the University of North Carolina Alumni Heart Study (UNCAHS), a large, nonclinical longitudinal study of adults (for details, see Hooker, Hoppmann, \& Siegler, 2010) that includes measures of lifetime trauma exposure (Ogle, Rubin, \& Siegler, 2013) and an archive of personality data (Costa, Herbst, McCrae, \& Siegler, 2000; Siegler et al., 1992). UNCAHS respondents who completed the NEO Personality Inventory (NEO; Costa \& McCrae, 1992) at two points in midlife (approximately eight years apart) and later reported their lifetime exposure to traumatic events were grouped according to the timing of their traumas. The first subset included individuals who experienced all of their lifetime traumas in the interval between the two NEOs $(n=60)$. Because trauma exposure could have affected only the second personality assessment for these individuals, examination of the difference in pre- and post-trauma neuroticism scores provided the most sensitive within-subjects investigation of potential change in neuroticism following trauma exposure. The second subset included individuals who were exposed to all of their lifetime traumas before the first personality assessment $(n=455)$. To the extent that neuroticism increases following trauma exposure, neuroticism scores on the first NEO were expected to be higher for these individuals compared to participants who had not yet experienced traumatic events at the time of the first NEO. The third subset included individuals who experienced all of their traumas after the second personality assessment $(n=155)$. For these individuals, exposure to traumatic events could not have affected neuroticism scores on either NEO. If neuroticism is elevated following exposure to traumatic events, then neuroticism should be lower on the first and second NEO for these participants compared to the other two participant subsets.
Although we were primarily interested in change in neuroticism among these three participant subsets, we also examined longitudinal change in neuroticism among individuals who reported no traumatic events $(n=289)$ and among all members of the UNCAHS for whom complete personality data were available $(n=3,257)$. Analyses using data from the total UNCAHS sample were conducted to examine the normative change in neuroticism during middle adulthood irrespective of exposure to trauma. In previous research with the UNCAHS sample (Costa et al., 2000), modest declines in midlife neuroticism were reported, consistent with other cross-sectional and longitudinal studies showing slow decreases in neuroticism throughout adulthood (Schaie, 2005; Terracciano, McCrae, Brant, \& Costa, 2005).

A second aim of our study was to extend prior research by investigating change in neuroticism following exposure to the type of traumatic events required for a diagnosis of PTSD according to the Diagnostic and Statistical Manual of Mental Disorders A1 criterion (DSM-IV-TR; American Psychiatric Association, 2000), which specifies that an individual must have experienced, witnessed, or confronted an event or events that involved actual or threatened death or serious injury, or a threat to the physical integrity of self or others. Lifethreatening events may have a more pronounced influence on neuroticism than the non-life-threatening negative events examined in previous research, which in general may not be extreme enough to alter personality. Furthermore, clustering multiple events together irrespective of their severity may obscure the impact of more severe traumas. Accordingly, a second set of analyses concentrated on change in neuroticism following single, life-threatening traumas.

In addition to measuring domain-level neuroticism, we also assessed change in the lower-level indices of neurotic traits. Few studies have examined the impact of exposure to traumatic or stressful life events on longitudinal changes in neuroticism at the facet level (e.g., Costa et al., 2000; Sutin, Costa, Wethington, \& Eaton, 2010). In one notable exception, Löckenhoff et al. (2009) found that individuals who reported recent adverse life events (e.g., divorce, job loss, death of a loved one) exhibited increases in domain-level neuroticism as well as facet-level angry hostility and depression scores compared to the rest of the sample. Investigating the individual neurotic traits that may be impacted by exposure to trauma may further advance our understanding of the specific ways in which personality is altered by traumatic life experiences.

Finally, in supplemental analyses, we examined NEO neuroticism and neuroticism as measured by the Minnesota Multiphasic Personality Inventory (MMPI) upon entering college for individuals who experienced early life traumas. Associations between childhood abuse and personality dimensions, such as hostility and neuroticism, have been reported in previous research with substance-dependent patients (Roy, 2001, 2002). In the present study, we extended this research to a nonclinical sample by examining differences in adulthood neuroticism among individuals with recent severe traumas com- 
pared to those who experienced severe traumas early in life. Longitudinal change in midlife neuroticism was also assessed for this group.

\section{METHOD}

\section{Participants and Procedure}

Participants were drawn from the UNCAHS, an ongoing longitudinal study of adults that was originally designed to examine the impact of personality on coronary heart disease. Details of the UNCAHS have been described previously (Hooker et al., 2010; Siegler et al., 1992). Personality assessments were completed by mail in 1988-1991 and in 19971998 (see Siegler et al., 1990). Data concerning lifetime trauma exposure were obtained in 2008-2009 (Wave 12). UNCAHS members first received instructions to complete the Wave 12 questionnaire online. Individuals who did not respond were mailed an identical paper version. All procedures were approved by the Duke University Medical Center institutional review board.

UNCAHS respondents who completed both NEOs and reported their lifetime exposure to traumatic events were included in the initial sample $(n=2,728)$. Participants were excluded from our primary analyses if they experienced traumas that were too widely distributed across the life course to test our hypothesis (e.g., having one trauma before the first NEO and one trauma after the second NEO; $n=1,668)$, did not report their age at one or more traumas $(n=101)$, or reported zero traumatic events $(n=289)$. The remaining individuals were considered eligible for inclusion in our primary analyses because they completed both administrations of the NEO, reported their lifetime exposure to traumatic events, and experienced all of their lifetime traumas before, between, or after the two NEOs $(n=670)$. For this group, the mean age at the first and second administration of the NEO was $41.61(S D=$ $2.93)$ and $49.74(S D=2.80)$, respectively. The mean age at the 12 th wave of data collection was $60.55(S D=2.79)$. Participants were predominantly male $(65.22 \%)$ and Caucasian $(99.70 \%)$. Approximately $8 \%$ had less than a college degree, $23.28 \%$ had a bachelor's degree, $23.58 \%$ had a bachelor's degree plus additional training, $25.67 \%$ had a master's degree, and $18.81 \%$ had a doctorate or medical degree. The median household income reported in 2001-2002 was in the \$7099,999 range. Because the UNCAHS sample is not representative of the general population, sociodemographic variables were included as covariates in our analyses.

To examine change in neuroticism following exposure to life-threatening traumas only, a second set of analyses included UNCAHS respondents whose single most distressing trauma met the A1 PTSD diagnostic criterion. Of the 2,728 UNCAHS respondents who completed both NEOs and reported their lifetime exposure to traumatic events, participants were excluded if they reported zero traumatic events $(n=289)$, they did not nominate an event that currently caused them distress $(n=320)$ and date the event $(n=487)$, their currently most distressing trauma did not meet the A1 criterion $(n=795)$, or their A1 trauma occurred the same year that they completed one of the NEOs $(n=30)$, which precluded the ability to determine the temporal order of the life-threatening trauma in relation to completion of the NEO. The remaining 807 individuals were included in our analyses concerning lifethreatening trauma exposure. The gender, ethnicity, education, and income information for these participants was nearly identical to the information reported for participants included in our primary analyses.

\section{Measures}

Traumatic Events. The Traumatic Life Events Questionnaire (TLEQ; Kubany et al., 2000) assesses lifetime exposure to 23 types of traumatic events (e.g., sexual assault, warfare or combat, natural disaster). For each type, individuals indicated how often the event occurred, whether or not their experience met the DSM-IV-TR A1 (i.e., experienced, witnessed, or confronted an event that involved actual or threatened death or injury, or a threat to the physical integrity of the self or others) and A2 (i.e., experienced intense fear, helplessness, or horror during or immediately after the event) diagnostic criteria (American Psychiatric Association, 2000), as well as their age at the most severe occurrence of each event type. Participants were also asked to nominate one event that currently bothered them most. The TLEQ has strong psychometric properties, including high convergent validity with structured clinical interviews (Kubany et al., 2000). Similar to epidemiological studies of trauma exposure that employed mail surveys (e.g., Kessler et al., 1995), modifications were made to the TLEQ to provide respondents with extra privacy in reporting and to reduce redundancy (for details, see Ogle et al., 2013).

Neuroticism. Neuroticism was measured twice using the NEO Personality Inventory (NEO-PI; for details, see Costa et al., 2000). Raw scores were converted to $t$-scores based on adult combined-sex norms. Domain and facet scores were analyzed. The NEO-PI has strong psychometric properties (Cronbach's $\alpha=.92$ ) and is well validated (Costa \& McCrae, 1992).

A subset of participants also completed the MMPI upon entering college in 1964-1965 $\left(M_{\text {age }}=18.28, S D=1.69\right)$. Factor-analyzed neuroticism scores derived from the MMPI (see Costa, Zonderman, McCrae, \& Williams, 1985; Siegler et al., 1990) were included in supplemental analyses. The neuroticism factor has good internal consistency (Cronbach's $\alpha=.92$ ).

\section{RESULTS}

\section{Descriptive Analyses}

The prevalence of exposure to traumatic events varied widely across event type. Over one-third of participants (36.72\%) 
included in our primary analyses reported the sudden or unexpected death of a loved one. Other high-frequency events included non-live birth pregnancies (28.81\%), life-threatening illnesses or accidents of loved ones (20.30\%), car accidents $(16.87 \%)$, and other life-threatening events $(15.25 \%)$. In addition, $12.87 \%$ experienced a life-threatening personal illness or accident, $11.79 \%$ reported sexual assaults, $10.33 \%$ reported death threats, $10.19 \%$ witnessed childhood family violence, $8.21 \%$ were exposed to warfare or combat, $6.87 \%$ reported other accidents with injury, $6.45 \%$ reported armed robberies, $5.97 \%$ reported physical assault by a partner, $4.19 \%$ witnessed an attack or a murder, $4.18 \%$ were physically assaulted by a stranger, $4.05 \%$ reported childhood physical punishment, $4.04 \%$ were stalked, $4.03 \%$ experienced natural disasters, and $2.84 \%$ elected to not describe their traumatic experiences. Intercorrelations among the sociodemographic variables and neuroticism scores revealed small negative associations between age and NEO 1 scores $(r=-.10, \mathrm{p}<.01)$, between education and NEO 1 scores $(r=-.09, \mathrm{p}<.05)$, and between household income and NEO 1 and 2 scores $(r=-.12, \mathrm{p}<.01$ and $r=-.09, \mathrm{p}<.05$, respectively). Females reported significantly higher neuroticism scores on the NEO $1(M=53.07, S D$ $=10.63)$ and the NEO $2(M=51.44, S D=10.57)$ compared to men (NEO 1: $M=46.84, S D=9.82$; NEO 2: $M=45.75, S D=$ $10.01), F_{\mathrm{s}}(1,668)>47.18, \mathrm{p}<.001$, Cohen's $d>.55$. These findings are consistent with previous reports of differences in personality stability across demographic groups (e.g., Löckenhoff et al., 2008). Given these intercorrelations and gender differences, our primary analyses were adjusted for age, education, gender, income, as well as the number of years between the first and second NEO $(M=8.14, S D=1.85)$.

\section{Changes in Neuroticism Following Trauma Exposure}

We first present results from an omnibus repeated-measures ANCOVA that simultaneously examined the longitudinal change in neuroticism for the three participant subsets (those who encountered all of their traumas in the interval between the NEOs, those who experienced all of their traumas before the first NEO, and those who experienced all of their traumas after the second NEO). Means (SDs) are reported in the top section of Table 1. No significant main effect of time or group emerged, $F(1,662)=2.16$ and $F(2,662)=.44$, respectively. The Time $\times$ Group interaction was also nonsignificant, as reflected by the approximately parallel slopes in neuroticism for the three participant subsets depicted in Figure 1, $F(2,662)$ $=.46$. In contrast to the expected result if neuroticism increases following trauma exposure, this analysis indicates that for participants who encountered all of their traumas in the years between the two personality assessments, neuroticism scores on the first and second NEO did not significantly change. Similarly, the between-subjects comparisons of the impact of trauma exposure on neuroticism also revealed no differences across groups. Participants who experienced all of their lifetime traumas before the first NEO did not have significantly higher NEO 1 scores compared to participants without histories of trauma at the time of the first NEO. Likewise, on the second NEO, participants who had not yet been exposed to traumatic events failed to exhibit lower neuroticism scores compared to participants with histories of exposure. When the analysis was repeated with covariates removed, only the main effect of time was significant, $F(1,667)=13.24, \mathrm{p}<.001, \eta_{p}{ }^{2}$ $=.02$. Although the effect size was small, overall neuroticism scores declined over time.

A second repeated-measures ANCOVA was conducted to examine change in neuroticism following exposure to lifethreatening traumas among participants who reported that their most distressing trauma met the A1 PTSD diagnostic criterion $(n=807)$. The number of years between participants' A1 trauma and the subsequent NEO (NEO 2 for the third group) was added as a covariate to the model. Means (SDs) are presented in the middle section of Table 1. Consistent with the results from the first ANCOVA, the main effects of time and group were nonsignificant, as was the Time $\times$ Group interaction, $F_{\mathrm{s}}(1-2,798)<2.86$. Neuroticism scores did not signifi-

Table I Means (SDs) and Rank-Order Stability of NEO Neuroticism Scores

\begin{tabular}{lclll}
\hline Subsamples & $n$ & NEO I & NEO 2 & $r($ NEO I - NEO 2) \\
\hline All traumas before NEO I & 455 & $49.98(10.6 \mathrm{I})$ & $47.84(10.73)$ \\
All traumas between NEOs & 60 & $49.5 \mathrm{I}(9.83)$ & $48.45(9.94)$ & .79 \\
All traumas after NEO 2 & 155 & $48.89(10.62)$ & $47.11(10.28)$ & .76 \\
Total & 670 & $49.01(10.53)$ & $47.73(10.55)$ & .81 \\
Life-threatening trauma before NEO I & 387 & $49.74(10.80)$ & $48.96(10.94)$ & .79 \\
Life-threatening trauma between NEOs & 95 & $47.78(9.10)$ & $47.97(8.96)$ & .78 \\
Life-threatening trauma after NEO 2 & 325 & $49.56(10.74)$ & $47.77(10.44)$ & .70 \\
Total & 807 & $49.44(10.60)$ & $48.36(10.53)$ & .79 \\
No traumatic events & 289 & $48.3(9.91)$ & $47.32(9.30)$ & .79 \\
All UNCAHS respondents & 3,257 & $49.78(10.91)$ & $48.62(10.60)$ \\
\hline
\end{tabular}

Note. Means were adjusted for sociodemographic variables, including age, education, gender, and income, as well as the test-retest interval. The number of years between participants' life-threatening trauma and the subsequent NEO (NEO 2 for the third group) was added as an additional covariate to the analyses concerning life-threatening traumas. 

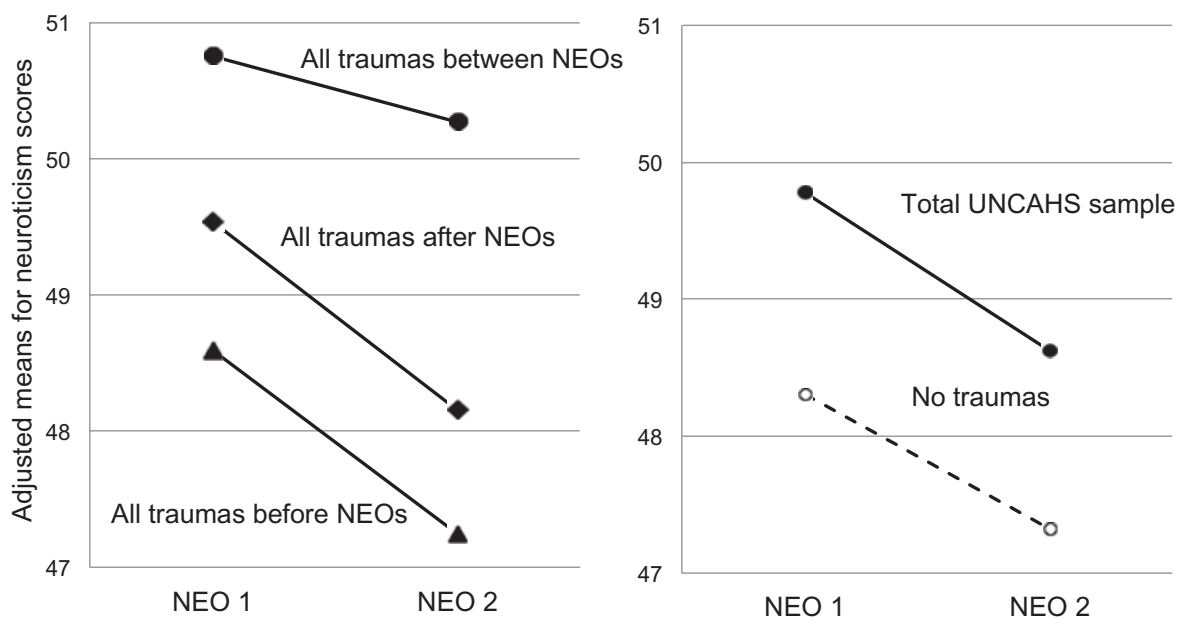

Figure I The left panel displays adjusted means for neuroticism scores from the first and second NEO Personality Inventory as a function of the timing of participants' exposure to all reported traumatic events. The right panel displays the adjusted means for neuroticism scores for participants who reported no traumatic events $(n=289)$ compared to all UNCAHS respondents who completed both NEOs $(n=3,257)$.

cantly change over time for participants who experienced their most distressing life-threatening trauma before, between, or after the two administrations of the NEO. These findings suggest that midlife neuroticism was not significantly elevated following exposure to severe traumas when analyses were adjusted for relevant covariates.

When covariates were omitted from the model, however, a significant main effect of time emerged, which was qualified by a significant Time $\times$ Group interaction, $F(1,804)=6.93$, $\mathrm{p}<.01, \eta_{p}{ }^{2}=.01$ and $F(2,804)=3.45, \mathrm{p}<.05, \eta_{p}{ }^{2}=.01$, respectively. Simple effects analyses revealed a weak trend in the expected direction. Consistent with previous reports of normative age-related declines in neuroticism during adulthood, modest decreases were found in midlife neuroticism for individuals who reported exposure to life-threatening traumas capable of producing PTSD before or after the NEOs, $F(1$, $386)=4.52, \mathrm{p}<.05, \eta_{p}{ }^{2}=.01$ and $F(1,324)=21.53, \mathrm{p}<.001$, $\eta_{p}{ }^{2}=.06$, respectively. In contrast, neuroticism remained stable for individuals who reported exposure to an A1 trauma in the interval between the two personality assessments.

To examine change in midlife neuroticism for individuals without histories of trauma exposure, neuroticism scores from the first and second NEO were compared for participants who reported no traumatic events on the TLEQ (dashed line, right panel of Figure 1). No significant difference emerged when results were adjusted for covariates, $F(1,283)=2.09$. However, when covariates were omitted from the model, the decline in neuroticism from the first to the second NEO reached significance, $F(1,288)=7.13, \mathrm{p}<.01, \eta_{p}{ }^{2}=.02$.

The final comparison group consisted of all UNCAHS respondents who completed both NEOs (solid line, right panel of Figure 1). A repeated-measures ANCOVA indicated that the decline in neuroticism scores over time was not significant, $F(1,3251)=.02$. The difference, however, reached signifi- cance when covariates were omitted from the model, $F(1$, $3256)=95.11, \mathrm{p}<.001, \eta_{p}{ }^{2}=.03$.

Longitudinal Stability. To examine whether exposure to trauma influenced the rank-order consistency of neuroticism, correlation coefficients of neuroticism scores from the first and second NEO were first calculated for each subgroup (Table 1). The correlations ranged from .70 to $.81(p \mathrm{~s}<.001)$. Next, tests of the significance of the differences between correlations were computed using Fisher $r$-to- $z$ transformation. The stability of neuroticism for individuals who experienced a lifethreatening trauma between the two NEOs was significantly lower than the stability of neuroticism for all UNCAHS respondents $(z=2.19, \mathrm{p}<.05)$. No other significant differences were found. In general, these results complement those concerning mean-level changes in neuroticism by showing that the rank-order consistency of neuroticism is comparable among individuals with and without histories of trauma. In addition, the finding for individuals exposed to A1 traumas between the NEOs provides modest support for the view that severe traumas may alter the stability of midlife neuroticism.

Neuroticism Facets. To examine possible changes in facetlevel neuroticism traits following trauma exposure, repeatedmeasures ANOVAs were conducted for the six neuroticism facets: anxiety, angry hostility, depression, self-consciousness, impulsiveness, and vulnerability to stress. Models were tested with and without covariates for the all-traumas subgroup and the life-threatening traumas subgroup to parallel the analyses reported for domain-level neuroticism. The alpha level was adjusted to $\mathrm{p} \leq .008$ to account for multiple comparisons.

Although negative trends over time were found for all facets in models with and without covariates, few significant effects emerged. Only the self-consciousness facet in the life- 
threatening traumas subgroup (Time 1: $M=49.10, S D=10.11$; Time 2: $M=48.26, S D=10.14$ ) and the impulsiveness facet in the all-traumas subgroup (Time $1: M=50.24, S D=10.37$; Time 2: $M=49.19, S D=10.49)$ significantly declined over time, $F(1,804)=8.62, \mathrm{p}<.008, \eta_{p}{ }^{2}=.01$ and $F(1,662)=8.22$, $\mathrm{p}<.008, \eta_{p}^{2}=.01$, respectively. The differences were not significant, however, in both models with and without covariates. In addition, a significant main effect of group emerged for the angry hostility facet in the life-threatening traumas subgroup, $F(2,804)=7.10, \mathrm{p}<.001, \eta_{p}{ }^{2}=.02$. Pairwise comparisons indicated that individuals who experienced their most distressing life-threatening trauma before the first NEO had higher hostility scores $(M=51.19, S D=10.04)$ than those who experienced their most distressing life-threatening trauma after both NEOs $(M=48.71, S D=9.60)$. However, the difference attenuated to nonsignificant levels with covariates in the model. Importantly, none of the interactions were significant for the all-traumas subgroup or the life-threatening traumas subgroup. In sum, results from the facet analyses mirrored those from the domain-level analyses and are consistent with previous reports of normative age-related declines in neuroticism observed across adulthood.

\section{Early Life Traumas}

Given that participants were approximately 42 and 50 years of age when the two NEOs were completed, the results reported thus far are limited to change in neuroticism following traumatic events encountered during middle adulthood. To examine adulthood neuroticism among individuals exposed to traumatic events earlier in the life course, participants who experienced life-threatening traumas prior to the first NEO were further divided into four subsets according to the developmental period of their trauma: childhood (1-12 years of age, $n=54)$, adolescence (13-18 years of age, $n=37)$, young adulthood (19-34 years of age, $n=235$ ), and middle adulthood (35- age at first NEO, $n=61$ ). An ANCOVA that compared neuroticism scores from the first NEO among the four groups while controlling for sociodemographic variables revealed a significant main effect of developmental period, $F(3,379)=3.83, \mathrm{p}<.01, \eta_{p}{ }^{2}=.03$ (solid line, Figure 2). Pairwise comparisons indicated that participants who experienced a life-threatening trauma during childhood had significantly higher neuroticism scores $(M=$ $52.73, S D=11.64)$ compared to individuals who experienced a life-threatening trauma during young $(M=49.03, S D=10.21)$ or middle adulthood $(M=47.96, S D=9.94)$. Neuroticism scores for the adolescent exposure group $(M=52.85, S D=$ 12.72) were also significantly higher than those for the young and middle adulthood groups. These results indicate that individuals who experienced severe traumas early in life have significantly higher levels of neuroticism in midlife compared to individuals who experienced recent severe traumas.

To examine change in midlife neuroticism for individuals who experienced a life-threatening trauma early in the life course, a repeated-measures ANCOVA was conducted that compared neuroticism scores from the NEO 1 and 2 for individuals who experienced a life-threatening trauma prior to the first NEO. A significant main effect of group emerged, $F(3$, $378)=3.82, \mathrm{p}<.05, \eta_{p}{ }^{2}=.03$. Pairwise comparisons revealed that individuals who experienced a life-threatening trauma in childhood had higher neuroticism scores $(M=52.69, S D=$ 11.31) than individuals who experienced a life-threatening trauma in middle adulthood $(M=47.39, S D=9.10)$. Similarly, individuals who experienced a life-threatening trauma in ado-

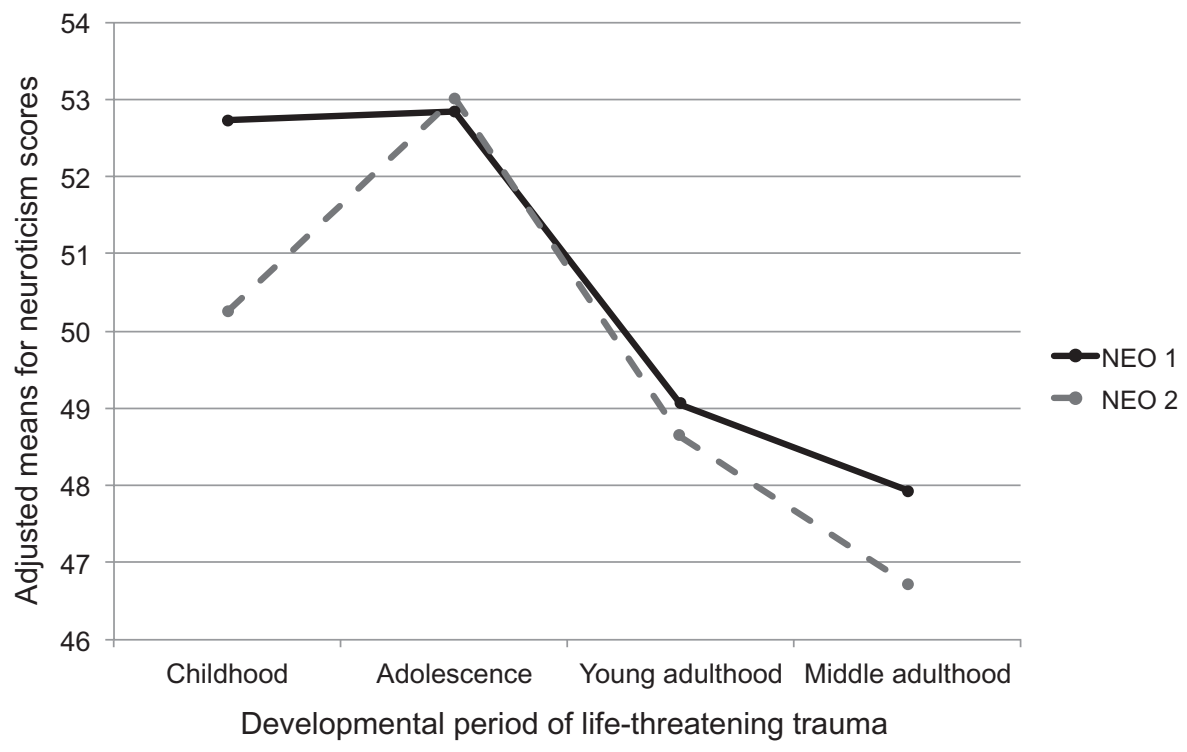

Figure 2 Adjusted means for neuroticism scores from the first and second NEO as a function of the developmental period of participants' currently most distressing life-threatening trauma. Childhood = traumas at ages I to I2 $(n=54)$, adolescence $=$ traumas at ages I 3 to $I 8(n=37)$, young adulthood $=$ traumas at ages 19 to $34(n=235)$, and middle adulthood = traumas at age 35 through the age when the first NEO was completed $(n=61)$. 
lescence had higher neuroticism scores $(M=52.46, S D=$ 11.86) than individuals who experienced such traumas in young $(M=48.59, S D=9.77)$ or middle adulthood.

In the model with covariates omitted, neuroticism scores declined over time (NEO 1: $M=49.74, S D=10.80$; NEO 2: $M$ $=48.96, S D=10.94), F(1,383)=5.49, \mathrm{p}<.05, \eta_{p}{ }^{2}=.01$. The main effect of group was also retained, $F(3,383)=4.40$, $\mathrm{p}<$ $.01, \eta_{p}{ }^{2}=.03$. In addition to the significant pairwise comparisons that emerged in the model with covariates, individuals who experienced a life-threatening trauma in childhood had higher neuroticism scores than those who experienced such traumas in young adulthood. Overall, these findings are consistent with the analysis of NEO 1 scores in showing that individuals exposed to severe traumas earlier in the life course exhibited higher levels of midlife neuroticism than individuals exposed to trauma after the transition to adulthood. In addition, as revealed by the absence of Time $\times$ Group interactions, no evidence of differences in the longitudinal change of midlife neuroticism was found among individuals who experienced severe traumas during childhood, adolescence, young adulthood, and middle adulthood.

Additional exploratory analyses were conducted using college-age MMPI scores that were available for approximately $79 \%(n=306)$ of this group. In chi-square and $t$ tests that compared participants who did and did not complete the MMPI, no differences emerged on key demographic characteristics, including age, income, and education, $t(385)<.44$, $\chi^{2}(4, n=387)=2.09$. However, comparison of the gender distributions revealed that the group who completed the MMPI had a greater proportion of males, $\chi^{2}(1, n=387)=19.6, \mathrm{p}<$ .001 . Factor-analyzed neuroticism scores derived from the MMPI were compared for participants whose life-threatening trauma occurred during childhood (ages $1-12, n=45$ ) versus adolescence (ages 13-age at MMPI, $n=22$ ). Although traumatic events occurred on average $10.59(S D=3.68)$ years before the MMPI for the childhood group and $2.96(S D=1.94)$ years before the MMPI for the adolescent group, the childhood exposure group had average college-age neuroticism scores that were approximately five points higher $(M=29.10, S D=$ $12.95)$ than the adolescent exposure group $(M=23.76, S D=$ 9.50 ). This finding provides convergent validity for the results from the NEO, which showed that individuals with early life traumas exhibit higher levels of adulthood neuroticism compared to those who experienced recent traumatic events.

\section{DISCUSSION}

Using a longitudinal design, the present study examined change in midlife neuroticism following exposure to traumatic events in a large nonclinical sample of adults. Results indicated that neuroticism is relatively stable across middle adulthood among individuals with and without histories of trauma exposure. Specifically, no differences were found in pre- and post-trauma neuroticism scores among individuals who experienced all of their traumatic life events in an 8-year interval between two assessments of personality during middle adulthood. Analyses did not vary with and without sociodemographic factors and the test-retest interval controlled. In addition, no differences in midlife neuroticism scores were found among individuals who experienced all of their lifetime traumas before, between, or after the personality assessments. A comparable pattern of findings emerged when the analyses were limited to severe, life-threatening traumas. Furthermore, results from the facet-level analyses largely mirrored those for domain-level neuroticism. Overall, these findings suggest that midlife neuroticism does not reliably change following exposure to traumatic events.

Our finding that neuroticism is relatively stable following traumatic events encountered in middle adulthood is consistent with results from both cross-sectional and longitudinal studies concerning the normative developmental trajectory of adulthood personality. For example, in a longitudinal examination of personality development, Terracciano et al. (2005) found that neuroticism declined across adulthood at a relatively slow rate of less than $1 t$-score per decade until approximately age 80. Modest mean-level declines in neuroticism across the adult life span have also been found in studies of national probability samples (Mroczek \& Spiro, 2003) and in cross-cultural research (McCrae et al., 1999). In general, these findings support essentialist theories of personality development, according to which personality is biologically determined and is therefore relatively immune to environmental change (McCrae et al., 2000).

Our results are also consistent with previous reports of the relative stability of adulthood personality following adverse life events. In a longitudinal study of approximately 14,000 individuals in Germany, Specht et al. (2011) examined the impact of major life events on personality across a 4-year period of adulthood. Decreases in neuroticism (i.e., emotional instability) emerged only for women who reported moving out of their parental home. No evidence was found for aggregated or specific negative life events (e.g., separation from a partner, divorce, death of a spouse, parental death, or unemployment) to impact the mean-level or rank-order stability of neuroticism. Similarly, in a previous report of findings from UNCAHS respondents (Costa et al., 2000), exposure to stressful events did not influence personality change during middle adulthood, with the exception of divorce and employment loss. However, individuals who reported perceptions of negative changes in several domains of life (i.e., social, work, family, and economic status) exhibited small increases in domain-level neuroticism and in select neuroticism facets (i.e., anxiety, depression, vulnerability) compared to individuals who reported stability in these areas of life. Sutin et al. (2010) also found that reported exposure to traumatic events did not predict changes in midlife personality, but that individuals who described their most stressful experience to be a point at which their lives changed for the worse increased in both domain-level neuroticism as well as their vulnerability to feelings of shame and embarrassment (N4 self-consciousness) 
across middle adulthood. Collectively, these findings suggest that the subjective interpretation of negative life events may have a greater impact on personality than the experience of the event itself.

It is important to note, however, the few aspects of our results for individuals who experienced severe, life-threatening traumas that may be interpreted as modest evidence of personality change following traumatic events. First, in contrast to the well-established normative declines in neuroticism that are typically observed throughout adulthood (e.g., McCrae et al., 1999; Mroczek \& Spiro, 2003; Terracciano et al., 2005), midlife neuroticism scores remained stable for individuals exposed to A1 traumas in the interval between the two NEOs. Second, although the magnitudes of the rank-order correlations were similar to those reported in previous studies (e.g., Terracciano, Costa, \& McCrae, 2006), the rank-order consistency of neuroticism was lower for individuals who experienced life-threatening traumas between the personality assessments compared to the other subgroups in the sample. Finally, consistent with previous reports of increases in angry hostility following exposure to adverse life events (Löckenhoff et al., 2009), individuals who experienced severe, life-threatening traumas before the personality assessments reported higher angry hostility facet scores compared to individuals exposed to severe traumas after the personality assessments. Taken together, these findings suggest that exposure to traumatic life events may alter the normative developmental trajectory of adulthood neuroticism. However, it is important to note that the effect sizes for these results were small and that the findings concerning mean-level changes did not hold when sociodemographic covariates were included in the models.

Additional support for the view that traumatic experiences may affect the developmental course of neuroticism may be drawn from our supplemental analyses concerning traumas encountered early in the life course. Specifically, we found that individuals who experienced severe traumatic events during childhood and adolescence exhibited higher midlife neuroticism compared to individuals who experienced such traumas in young or middle adulthood. An exploratory examination of college-age MMPI scores showed that individuals who experienced severe childhood traumas also exhibited higher neuroticism in young adulthood than individuals who experienced recent traumatic events prior to college entry. Thus, the elevated levels of neuroticism exhibited by individuals with severe childhood traumas upon college entry were evident approximately 20 years later. Collectively, these results suggest that life-threatening events encountered before the transition to adulthood may exert a greater impact on adulthood personality than recent traumatic experiences that occur in adulthood. Although our study did not examine mechanisms that may promote the enduring nature of this pattern of elevation, one possibility is that severe early life traumas may play a formative role in shaping the developmental course of neuroticism through their accentuating effect on preexisting individual differences in personality. When interpreted within the frame- work of the accentuation model of personality coherence (Caspi \& Moffitt, 1993), early life traumatic experiences may have exacerbated or reinforced the preexisting neurotic tendencies of individuals exposed to severe early life traumas relative to their non-trauma-exposed peers. The pronounced neurotic tendencies of individuals with histories of childhood trauma may have been sustained over time and across contexts through various forms of person-environment interactions (see Fraley \& Roberts, 2005). Additional prospective research is needed to further investigate longitudinal changes in personality following early life trauma exposure.

\section{STRENGTHS AND LIMITATIONS}

Strengths of the current study include a large sample, a longitudinal design, and two assessments of neuroticism measured approximately eight years apart using a well-validated personality test. In contrast to previous studies concerning personality change following adverse events, our sample was divided according to the timing of participants' lifetime exposure to traumatic events. Thus, we provide the most sensitive test to date of changes in neuroticism following trauma exposure. Furthermore, data concerning personality and lifetime trauma exposure were collected independently (approximately 10 years apart), which limited any potential bias resulting from trauma-related distress on the measures of neuroticism. However, several limitations of the study should be noted. First, the age distribution of traumatic events in relation to participants' age when personality was measured in the UNCAHS restricts our primary findings to the impact of traumas encountered in middle adulthood. Future longitudinal studies should aim to examine the impact of traumas experienced at other points during the life course on personality. In addition, the retrospective nature of the lifetime trauma exposure data may have resulted in a biased estimation of events. However, research regarding the validity of retrospective reports indicates that biases typically result in underreporting, at least for childhood adversities (e.g., Hardt \& Rutter, 2004). Third, some of the subgroups in our analyses concerning childhood traumas were relatively small. These findings therefore require replication. Finally, the generalizability of our findings may be limited by the selective nature of the UNCAHS sample. Although the UNCAHS is closely representative of the UNC student body in the 1960 s, the sample is not representative of the general U.S. population with respect to gender, education, and socioeconomic status. Despite this limitation, our findings appear to be consistent with previous studies concerning the longitudinal course of personality development in middle adulthood.

\section{CONCLUSIONS}

Overall, our results demonstrate that neuroticism in middle adulthood is relatively stable following exposure to traumatic 
events. No differences in the longitudinal stability of neuroticism were found for individuals who experienced all of their traumatic life events before, between, or after two assessments of personality completed in middle adulthood. Although modest evidence emerged in support of the view that severe, life-threatening traumas may alter the normative developmental trajectory of adulthood neuroticism, the results did not hold when covariates were included in the model. Our findings concerning individuals who experienced life-threatening traumatic events before the transition to adulthood suggest that childhood trauma exposure may be a risk factor for elevated levels of neuroticism in midlife.

\section{References}

American Psychiatric Association. (2000). Diagnostic and statistical manual of mental disorders (4th ed., text rev.). Washington, DC: Author.

Breslau, N., Davis, G. C., Andreski, P., \& Peterson, E. (1995). Risk factors for PTSD-related traumatic events: A prospective analysis. American Journal of Psychiatry, 152, 529-535.

Caspi, A., \& Moffitt, T. E. (1991). Individual differences are accentuated during periods of social change: The sample case of girls at puberty. Journal of Personality and Social Psychology, 61, 157168.

Caspi, A., \& Moffitt, T. E. (1993). When do individual differences matter? A paradoxical theory of personality coherence. Psychological Inquiry, 4, 247-271.

Costa, P. T., Herbst, J. H., McCrae, R. R., \& Siegler, I. C. (2000). Personality at midlife: Stability, intrinsic maturation, and response to life events. Assessment, 7, 365-378.

Costa, P. T., \& McCrae, R. R. (1992). Revised NEO Personality Inventory (NEO PI-R). Odessa, FL: Psychological Assessment Resources.

Costa, P. T., Zonderman, A. B., McCrae, R. R., \& Williams, R. B. (1985). Content and comprehensiveness in the MMPI: An item factor analysis in a normal adult sample. Journal of Personality and Social Psychology, 48, 925-933.

Fraley, R. C., \& Roberts, B. W. (2005). Patterns of continuity: A dynamic model for conceptualizing the stability of individual differences in psychological constructs across the life course. Psychological Review, 112, 60-74.

Hardt, J., \& Rutter, M. (2004). Validity of adult retrospective reports of adverse childhood experiences: Review of the evidence. Journal of Child Psychology \& Psychiatry, 45, 260-273.

Helson, R., Kwan, V. S. Y., John, O. P., \& Jones, C. (2002). The growing evidence for personality change in adulthood: Findings from research with personality inventories. Journal of Research in Personality, 36, 287-306.

Hooker, K., Hoppmann, C., \& Siegler, I. C. (2010). Personality: Life span compass for health. Annual Review of Gerontology and Geriatrics, 30, 201-232.

Kandler, C. (2012). Nature and nurture in personality development: The case of neuroticism and extraversion. Current Directions in Psychological Science, 21, 290-296.
Kessler, R. C., Sonnega, A., Bromet, E., Hughes, M., \& Nelson, C. B. (1995). Posttraumatic stress disorder in the National Comorbidity Survey. Archives of General Psychiatry, 52, 1048-1060.

Kubany, E. S., Leisen, M. B., Kaplan, A. S., Watson, S. B., Haynes, S. N., Owens, J. A., et al. (2000). Development and preliminary validation of a brief broad-spectrum measure of trauma exposure: The Traumatic Life Events Questionnaire. Psychological Assessment, 12, 210-224.

Lewis, M. (2001). Issues in the study of personality development. Psychological Inquiry, 12, 67-83.

Löckenhoff, C. E., Terracciano, A., Bienvenu, O. J., Patriciu, N. S., Nestadt, G., McCrae, R. R., et al. (2008). Ethnicity, education, and the temporal stability of personality traits in the East Baltimore Catchment Area study. Journal of Research in Personality, 42, 577-598.

Löckenhoff, C. E., Terracciano, A., Patriciu, N. S., Eaton, W. W., \& Costa, P. T. (2009). Self-reported extremely adverse life events and longitudinal changes in five-factor model: Personality traits in an urban sample. Journal of Traumatic Stress, 22, 53-59.

Magnus, K., Diener, E., Fujita, F., \& Pavot, W. (1993). Extraversion and neuroticism as predictors of objective life events: A longitudinal analysis. Journal of Personality and Social Psychology, 65, 1046-1053.

McCrae, R. R., Costa, P. T., Lima, M. P., Simoes, A., Ostendorf, F., Angleitner, A., et al. (1999). Age differences in personality across the adult life span: Parallels in five cultures. Developmental Psychology, 35, 466-477.

McCrae, R. R., Costa, P. T., Ostendorf, F., Angleitner, A., Hrebickova, M., Avia, M. D., et al. (2000). Nature over nurture: Temperament, personality, and life span development. Journal of Personality and Social Psychology, 78, 173-186.

Mroczek, D. K., \& Spiro, A. (2003). Modeling intraindividual change in personality traits: Findings from the Normative Aging Study. The Journals of Gerontology, 58B, 153-165.

Neyer, F. J., \& Asendorpf, J. B. (2001). Personality-relationship transaction in young adulthood. Journal of Personality and Social Psychology, 81, 1190-1204.

Ogle, C. M., Rubin, D. C., \& Siegler, I. C. (2013). The impact of the developmental timing of trauma exposure on PTSD symptoms and psychosocial functioning among older adults. Developmental Psychology. doi: 10.1037/a0031985.

Parslow, R. A., Jorm, A. F., \& Christensen, H. (2006). Associations with pre-trauma attributes and trauma exposure with screening positive for PTSD: Analysis of a community-based study of 2085 young adults. Psychological Medicine, 36, 387-395.

Roy, A. (2001). Childhood trauma and hostility as an adult. Psychiatry Research, 102, 97-101.

Roy, A. (2002). Childhood trauma and neuroticism as an adult: Possible implication for the development of common psychiatric disorders and suicidal behavior. Psychological Medicine, 32, 1471-1474.

Rubin, D. C., Berntsen, D., \& Bohni, M. K. (2008). A memory-based model of posttraumatic stress disorder: Evaluating basic assumptions underlying the PTSD diagnosis. Psychological Review, 115, 985-1011. 
Schaie, K. W. (2005). Developmental influences on adult intelligence: The Seattle Longitudinal Study. New York: Oxford University Press.

Siegler, I. C., Peterson, B. L., Barefoot, J. C., Harvin, S. H., Dahlstrom, W. G., Kaplan, et al. (1992). Using college alumni populations in epidemiologic research: The UNC Alumni Heart Study. Journal of Clinical Epidemiology, 45, 1243-1250.

Siegler, I. C., Zonderman, A. B., Barefoot, J. C., Williams, R. B., Costa, P. T., \& McCrae, R. R. (1990). Predicting personality from college MMPI scores: Implications for follow-up studies in psychosomatic medicine. Psychosomatic Medicine, 52, 644-652.

Specht, J., Egloff, B., \& Schmukle, S. C. (2011). Stability and change of personality across the life course: The impact of age and major life events on mean-level and rank order stability of the Big Five. Journal of Personality and Social Psychology, 101, 862-882.
Sutin, A. R., Costa, P. T., Wethington, E., \& Eaton, W. (2010). Turning points and lessons learned: Stressful life events and personality trait development across middle adulthood. Psychology and Aging, 25, 524-533.

Terracciano, A., Costa, P. T., \& McCrae, R. R. (2006). Personality plasticity after age 30. Personality and Social Psychology Bulletin, 32, 999-1009.

Terracciano, A., McCrae, R. R., Brant, L. J., \& Costa, P. T. (2005). Hierarchical linear modeling analyses of NEO-PI-R scales in the Baltimore Longitudinal Study of Aging. Psychology and Aging, 20, 493-506.

Vaidya, J. G., Gray, E. K., Haig, J., \& Watson, D. (2002). On the temporal stability of personality: Evidence for differential stability and the role of life experiences. Journal of Personality and Social Psychology, 83, 1469-1484. 